\title{
GPs' views on involvement of older patients: an European qualitative study
}

\author{
Raymond Wetzels $^{\mathrm{a}, *}$, Tina Aaen Geest ${ }^{\mathrm{b}}$, Michel Wensing ${ }^{\mathrm{a}}$, \\ Pedro Lopes Ferreira ${ }^{c}$, Richard Grol ${ }^{\mathrm{a}}$, Richard Baker ${ }^{\mathrm{d}}$ \\ a Centre for Quality of Care Research, UMC St. Radboud, P.O. Box 9101, 229 KWAZO, 6500 HB. Nijmegen. The Netherlands \\ 'Research Unit for General Practice, University of Aarhus, Denmark \\ ' Centre for Health Studies and Research, Faculty of Economics, University of Coimbra, Portugal \\ ${ }^{\mathrm{a}}$ Department of General Practice and Primary Health Care, Clinical Governance Research and Development Unit. University of Leicester, UK
}

Received 5 January 2003; received in revised form 21 March 2003; accepted 7 April 2003

\begin{abstract}
Involvement of older patients in general practice care is regarded as important, but is not widespread. To determine specific barriers to the involvement of older patients in general practice care and to identify variations between countries, we performed an international comparative study based on qualitative interviews with 233 general practitioners (GPs) in 11 countries. Most GPs thought that involving older patients had positive outcomes. GPs saw patient involvement as a process taking place solely during consultations. The main barrier for GPs was lack of time. Barriers related to older patients were their feelings of respect for doctors, their lack of experience in being involved and possible mental and physical impairments. To conclude, increasing involvement of older patients is not easy and will only be effective when GPs have adopted a more developed concept of patient involvement and are supported with the different methods for achieving this. The range of appropriate interventions may be similar in all countries.
\end{abstract}

(c) 2003 Elsevier Ireland Ltd. All rights reserved.

Keywords: Patient involvement; General practice; Older people

\section{Introduction}

In response to population ageing [1], health care policy emphasises the importance of involving older patients in the planning and delivery of healthcare $[2,3]$. However, older people may be reluctant to seek help for their complaints [4], they experience more difficulty in seeking and obtaining information during medical interviews $[5,6]$ and participate less in their consultations than other patients [7], even though they often have multiple health problems. Promoting involvement of older patients may improve the quality of care leading to better adherence with prescribed medication and advice provided [8], higher satisfaction with care and improvement in health status [9]. General practitioners (GPs) themselves think that involvement of patients is important [10]. Despite the different methods for involving patients [11], involvement of older patients is not widespread $[6,12]$. The reasons behind this contrast are unclear. Potential explanations are that GPs do not have enough time in consultations, they may lack concrete skills

\footnotetext{
${ }^{*}$ Corresponding author. Tel.: +31-24-3613114; fax: +31-24-3540166 E-mail address: r.wetzels@wok.umcn.nl (R. Wetzels).
}

or ideas for achieving involvement of their older patients, or they think that older patients do not want to be involved. The factors that influence effective implementation of involvement of older patients may depend on cultural factors and healthcare systems, and consequently there may be differences between countries [13]. In order to gain more insight into relevant barriers, we performed an international study, which aimed to identify GPs' thoughts of positive and negative consequences of involvement of older patients and the barriers and facilitators they perceive.

\section{Methods}

We performed a qualitative interview study in 11 countries, as part of a larger international project [14].

\subsection{Participants}

The participating countries were Austria, Belgium, Denmark, France, Germany, Israel, The Netherlands, Portugal, Slovenia, Switzerland and England. In order to obtain a typical cross-section of GPs, we sought a heterogeneous 
Table 1

Characteristics of GPs

\begin{tabular}{|c|c|c|c|c|c|c|c|c|}
\hline \multirow{2}{*}{ Country } & \multicolumn{2}{|l|}{ Age } & \multicolumn{2}{|l|}{ Gender } & \multicolumn{4}{|c|}{ Practice locality } \\
\hline & $<45$ & $>45$ & Male & Female & City & Urban & Rural & Total \\
\hline France & 5 & 11 & 12 & 4 & 5 & 8 & 3 & 16 \\
\hline Austria & 7 & 13 & 11 & 9 & 9 & 5 & 6 & 20 \\
\hline Belgium & 13 & 13 & 18 & 8 & 15 & 6 & 5 & 26 \\
\hline Switzerland & 6 & 14 & 14 & 6 & 9 & 3 & 8 & 20 \\
\hline Germany & 11 & 14 & 15 & 10 & 10 & 6 & 9 & 25 \\
\hline Israel & 11 & 11 & 12 & 10 & 12 & 4 & 6 & 22 \\
\hline Portugal & 12 & 11 & 9 & 14 & 10 & 8 & 5 & 23 \\
\hline Slovenia & 13 & 13 & 12 & 14 & 11 & 7 & 8 & 26 \\
\hline UK & 10 & 13 & 18 & 5 & 4 & 7 & 12 & 23 \\
\hline Denmark & 6 & 6 & 5 & 7 & 4 & 4 & 4 & 12 \\
\hline \multirow[t]{2}{*}{ The Netherlands } & 10 & 10 & 11 & 9 & 7 & 6 & 7 & 20 \\
\hline & $104(44,6 \%)$ & $129(55,4 \%)$ & $137(58,8 \%)$ & $96(41,2 \%)$ & $96(41,2 \%)$ & $64(27,5 \%)$ & $73(31,3 \%)$ & 233 \\
\hline
\end{tabular}

Nowadays we have patients who have to be responsible for their health. Therefore, it's not something imposed; instead, they have to know what's going on and they always have to participate in the decision, sometimes in the choice of the examinations and always in the decision of the treatment.

(Portugal, GP 4)

\subsection{Positive outcomes of patient involvement}

In all countries improvement of patients' acceptance of advice and adherence to medication was seen to be the most important expected positive outcome of patient involvement, along with improvements of doctor-patient relationships.

Table 2

Overview of themes

\begin{tabular}{|c|c|c|}
\hline Themes & $\begin{array}{l}\text { Number of countries in which the } \\
\text { theme was mentioned }(\max , 11)\end{array}$ & $\begin{array}{l}\text { Theme not present in the } \\
\text { following countries }{ }^{\mathrm{a}}\end{array}$ \\
\hline \multicolumn{3}{|l|}{ GPs' ideas of patient involvement } \\
\hline Explaining and informing patients about health aspects & 11 & \\
\hline Making decisions together with patients & 10 & $\mathrm{~F}$ \\
\hline Patients taking responsibility for their involvement & 10 & $\mathrm{~F}$ \\
\hline GPs giving patients the opportunity to ask questions & 9 & D, I \\
\hline \multicolumn{3}{|l|}{ Positive outcomes of patient involvement } \\
\hline Acceptance of advice and adherence to medication & 11 & \\
\hline Improved patient satisfaction & 11 & \\
\hline Improved GP satisfaction & 8 & B, I, S \\
\hline Equivalent division of responsibilities & 8 & $\mathrm{~B}, \mathrm{~F}, \mathrm{~N}$ \\
\hline \multicolumn{3}{|l|}{ Negative outcomes of patient involvement } \\
\hline Anxious towards patient involvement & 9 & $\mathrm{~B}, \mathrm{~F}$ \\
\hline \multicolumn{3}{|l|}{ Barriers of patient involvement } \\
\hline Organisational aspects (lack of time, and/or resources) & 11 & \\
\hline \multicolumn{3}{|l|}{ Patient related } \\
\hline Acceptance of authority & 8 & $\mathrm{~B}, \mathrm{~F}, \mathrm{P}$ \\
\hline Lack of familiarity with patient involvement or not wanting to be involved & 11 & \\
\hline Physical or cognitive impairments & 11 & \\
\hline \multicolumn{3}{|l|}{ GP related } \\
\hline Negative attitude towards patient involvement & 5 & $A, B, G, N, P, U$ \\
\hline Routine behaviour in daily practice & 7 & $\mathrm{~A}, \mathrm{~F}, \mathrm{~N}, \mathrm{P}$ \\
\hline \multicolumn{3}{|l|}{ Facilitators of patient involvement } \\
\hline More or other resources & 11 & \\
\hline Better prepared and informed patients & 10 & B \\
\hline GPs using communication skills & 11 & \\
\hline
\end{tabular}

\footnotetext{
${ }^{a}$ A, Austria; B, Belgium; D, Denmark; F, France; G, Germany; I, Israel; N, The Netherlands; P, Portugal; S, Slovenia; U, UK.
} 
I would hope patients are more likely to comply if they have the information and have an understanding and some stake in the management.

(UK, GP 13)

Almost all GPs thought, for example, that patient involvement would improve patient satisfaction, either by reducing patients' worries or increasing their understanding of disease and treatment options.

Many patients are much more satisfied if they can talk to the doctor because then also different types of fear or patients' viewpoints find expression.

(Slovenia, GP 02)

On the other hand, GPs' thought that their own satisfaction would improve as well. They saw patient involvement as a continuing challenge and thought it would give them more background information about patients, which would enable them to judge patients' needs and preferences better.

You are actually going to enjoy your practice more because the patients will understand your problems and you will understand their problems better

(UK, GP 7)

Finally, GPs mentioned a more equitable division of responsibilities during consultations as a result of involving patients.

People get a better control with their diabetes, probably a better regulation when they are involved. And if people themselves are in control of when they have to go to their yearly check-up and know what is going on at the check-ups, then we are two to remember what is going to happen.

(Denmark, GP 13)

\subsection{Negative outcomes of patient involvement}

Some GPs were anxious about patient involvement, for example, because consultations might be longer or people would ask (in the GPs' eyes) irrelevant questions.

I just don't want to educate my people in this sense. Then they start asking about all they have learned in the last TV show ['Consultation' by Dr. S. Stutz].

(Switzerland, GP 10)

\subsection{Perceived barriers to implementation of patient involvement}

Barriers to the implementation of patient involvement were mentioned at different levels. With regard to organisational aspects of practice, GPs commonly said they had insufficient time to involve patients. Also, lack of resources, such as money or personnel were mentioned as barriers.
GPs identified barriers relating to patients, such as cognitive and physical impairments. Also, acceptance of authority by older patients and their lack of familiarity with involvement or not wanting to be involved were labelled as barriers.

There are many who prefer being dominated by a doctor. ... Well, the younger patients accept my approach, but the older ones rather prefer instructions on what to do, with the general attitude: you are the doctor, you must know what I have to do...

(Austria, GP 14)

You have to stimulate older people more. By nature they are inclined not to ask too much, to agree with what the doctor says. They didn't learn well to be interactive.

(The Netherlands, GP 13)

Finally, besides their daily routine in the guidance of consultations, some GPs acknowledged their own attitude towards patient involvement as barriers.

[Encourage older patients] to speak about issues they did not raise before, I don't like it. It seems a little inquisitive to me (...) Organise meetings ... I don't think so. It sounds like wishful thinking.

(France, GP 3)

\subsection{Perceived facilitators to implementation of patient involvement}

GPs mentioned facilitators at different levels. As far as organisational aspects were concerned, more time, fewer patients per practice and more money would facilitate patient involvement.

In broad terms I would like to see patient involvement extend to political action as well. There needs to be more investment in primary care and the patients can play a part in that at the moment. Nobody is going to listen unless the patients say we think this is a good idea too

(UK, GP 9)

An occasional home visit by GPs was mentioned as a facilitator. At the individual level, informed and better prepared patients who are able to take responsibility, and GPs using specific communication skills would facilitate patient involvement in consultations.

Today people want to be better informed. Internet has such advantages, information can be retrieved and read without hurry. This may support and enhance the consultation process... and just by this bringing more impact to the consultation

(Switzerland, GP 4)

Some have little sheets of paper. They write down "this and that one came to my mind" and then we go through it bit by bit.

(Germany, GP 8) 


\subsection{International variation}

GPs in different countries had more or less consistent views on patient involvement (Table 2 ). They all saw advantages to patient involvement, although not always in the same area. The identification of barriers, especially GP related barriers, was somewhat different. In Denmark, France, Israel, Slovenia, Switzerland, some GPs mentioned their own negative attitude towards patient involvement as a barrier.

We probably are some pigheaded fellows from time to time, and we think things can only be done the way we already are doing them

(Denmark, GP 6)

GPs in all countries, except Austria, France, The Netherlands and Portugal, mentioned their own routine behaviour in daily practice as a barrier.

I had training in communication methods and I try to use the skills, but it is difficult in everyday practice. I have got difficulties by myself to put it into practice

(Germany, GP 22)

With regard to the facilitators of patient involvement, no major differences between countries emerged.

\section{Discussion and conclusion}

The most important findings from our study are the different barriers GPs perceive when enhancing involvement of older patients, their limited concept of patient involvement, their positive expectations of the outcomes of involvement of older patients and the limited variation between countries in the barriers and facilitators to the implementation of patient involvement.

\subsection{Discussion}

Firstly, the barriers to enhancing involvement of older patients are partly due to older patients themselves and partly to the organisation of primary care. The general prejudice of older people having difficulties becoming involved are confirmed by the GPs in our study. To judge whether this view is valid, it is crucial to know older patients' opinions about involvement, therefore, we interviewed patients of 70 years and older in another phase of the IMPROVE study, preliminary results show that patients think of involvement as an easy to access personal GP, whom they trust and who will adapt the consultation towards them as a patient(s) he knows. A review on patient preferences showed that older patients place greater emphasis on doctors making decisions and valued a more dominant doctor to a greater extent than younger patients [18]. Whether the views of the GPs makes them interact differently in consultations with older patients and, therefore, makes it more difficult for them to involve older patients, remains unclear. There are suggestions that they may do so, for example, in a study of patient-physician interactions for those 65 years or older, there was more chatting and less structuring, resulting in less counselling, health education and prevention [16]. Whether the organisation of practice, including lack of time and resources as well as the daily routine of GPs are genuine barriers that are hard to change, or whether they served as excuses for other barriers, such as reluctance to surrender some control did not become clear.

Secondly, when GPs were asked about their concept of patient involvement, they mainly referred to communication processes in the consultation, while methods to involve patients outside consultations were not mentioned. An explanation for this might be GPs' unfamiliarity with the methods to involve patients before or after consultations. These findings suggest that the broad concept of patient involvement, which is used in the scientific literature, is only understood in a relatively superficial way by GPs in Europe.

Thirdly, the positive outcomes GPs' expect from involving patients, such as improvement of adherence to medication and advice, are supported by findings in other empirical studies $[8,9,17]$. The fear of some GPs that consultations might become endless, may decrease when consultations are well structured and when practical tools are supplied which encourage older patients to take a more active role.

Finally, although it might be expected that barriers were influenced by the organisation of health systems or cultural factors, our comparison of barriers to implementation of patient involvement in 11 countries did not reveal clear differences between countries. The variation within countries was larger than the variation across countries. Possible explanations for this variation may be differences in socio-economic class, in cultural aspects and in spoken language between GPs and patients in some areas. This is confirmed by a recent literature review that shows that patient characteristics are an important determinant of preferences regarding primary care [18]. In addition, there may be differences in task profiles of GPs between urban and rural locations [19], different attitudes of GPs towards different problems of patients [20] and individual GP consultation style [21].

The main limitation of this study is related to the interpretation of the multi-lingual qualitative material. By using specific instructions, repeated checks of researchers in each country and a structured approach to analysis, we tried to reduce the influence of those problems as much as possible. Nevertheless, the international nature of this study precluded an in-depth analysis. The sample of GPs was purposefully sought amongst GPs of different gender, age and urbanisation level in order to explore a broad range of ideas. This international qualitative study was performed to gain an overview of the most important factors among GPs when considering involvement of older patients and should, therefore, be seen as exploratory. The results should not be generalised in a quantitative way. 